\title{
Interference Aware Channel Assignment Scheme in Multichannel Wireless Mesh Networks
}

\author{
Sunmyeng Kim \\ Department of Computer Software Engineering \\ Kumoh National Institute of Technology \\ Gumi, South Korea
}

\begin{abstract}
Wireless mesh networks (WMNs) are gaining significant attention to provide wireless broadband service. Nodes in a wireless mesh network can communicate with each other directly or through one or more intermediate nodes. Because of multi-hop transmissions with multiple contending and competing channels, performance of wireless mesh networks decreases. Supporting high performance is an important challenge in multi-hop mesh networks. Nassiri et al. proposed a Molecular MAC protocol for autonomic assignment and use of multiple channels to improve network performance. In the Molecular MAC protocol, nodes are either nuclei or electrons in an atom. Neighboring atoms use orthogonal channels to operate in parallel data transmissions. Each nucleus selects an idle channel that is not currently being occupied by its neighboring atoms with the assistance from electrons in the same atom. However, this protocol has the following drawback; since a nucleus allocates a channel with help from the electrons in its own transmission range, it is not able to recognize the existence of those atoms in the interference range. Therefore, allocating the same channel to neighboring atoms results in the deterioration of network performance. In order to resolve this problem, we propose a channel allocation scheme with the interference issue taken into account. Based on various simulation results, the proposed scheme was verified that different channels could be allocated to those neighboring atoms in the interference range.
\end{abstract}

Keywords-interference; channel assignment; multichannel; mesh network

\section{INTRODUCTION}

A wireless mesh network (WMN) is a cost-effective access network architecture. It is a promising wireless technology for numerous applications. It is gaining significant attention as a possible way for Internet service providers (ISPs) and carriers in order to provide wireless broadband service. In WMNs, nodes are consisted of mesh routers and mesh clients [1]. Mesh routers have minimal mobility and form the backbone of WMNs. When a node is within the transmission range of another node, they are considered as neighbors, and there is a wireless link between them. Some nodes, called gateways, are connected to the wired network, which connects the WMNs to rest of the Internet.

The packets sent by end users travel through wireless mesh networks over multiple hops. And gateway nodes relay traffic to and from the wired Internet. The performance of multi-hop communication quickly reduces as the number of hops becomes larger due to intra-flow and inter-flow interferences

This research was supported by Basic Science Research Program through the National Research Foundation of Korea (NRF) funded by the Ministry of Education, Science and Technology (2010-0025495).
[2-4]. This is because a wireless link is shared among neighboring nodes.

The intra-flow interference occurs because nodes are in each other's interference range along the path of the same flow. Nodes experience different amount of channel contention as shown in Fig. 1. Inter-flow interference occurs since multiple flows on the same channels between different routing paths operate and compete for the medium as shown in Fig. 2.

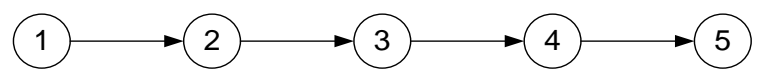

Fig. 1. Intra-flow interference

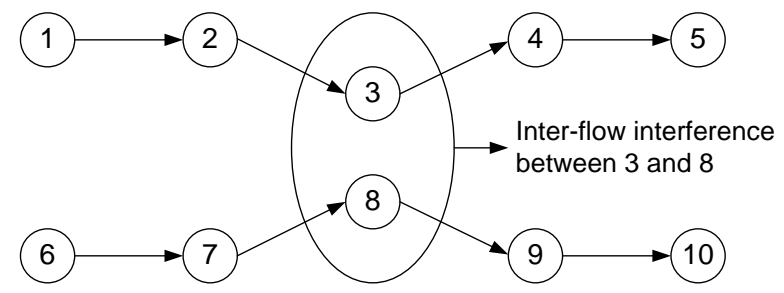

Fig. 2. Inter-flow interference

With a single channel, nodes operate on the same channel. Therefore, network performance decreases. Nodes can be equipped with multiple radios and channels. This means there is a unique frequency used for each wireless hop, and thus enables separation of wireless collision domain and reduces the interference and contention. This can significantly improve network performance without bandwidth degradation.

The design of the MAC protocol is the most likely challenge in WMNs. An interesting problem in WMNs is how to efficiently utilize multiple channels. Several MAC protocols for handling multiple channels have been proposed in the literature [5-17].

The algorithms proposed in [5] select channels for the mesh radios to minimize interference within the mesh network and between the mesh network and co-located wireless networks. A new method for the interference estimation is suggested. All available channels are periodically monitored by each mesh node and measured information of internal channel usage and external interference is shared with mesh nodes within 
interference range. Duarte et al. use game theory to design a systematic approach to utilize partially overlapped channels in WMNs while minimizing the adverse effect of adjacent channel interference [6]. In [7], both centralized and distributed algorithms are presented, which aim to minimize the number of pairs of links that are interfering. Molecular MAC protocol was proposed to organize the mesh network according to the molecular analogy [8-12]. It divides the network into atoms with nucleus nodes operating on fixed channels and electrons that dynamically switch channels between neighbor nuclei. Electrons can be shared by numerous atoms.

When a nucleus selects a channel, it receives help from the electrons in its own transmission range. Then, electrons deliver the information about those channels used by their parent atoms to the nucleus. Because an atom cannot be aware of the existence of other neighboring atoms if there are no electrons shared among them, the nucleus may allocate the same channel to such atoms. Although the proposed scheme naturally adopts a fundamental operation principle of the Molecular MAC protocol, it modifies a channel allocation method. The proposed scheme is somewhat similar to the Molecular MAC protocol method in that a channel is selected by the assistance of electrons. Then, a collision probability is applied to allocate a new channel in order to recognize any interference and avoid any overlapped channel allocation.

The paper is organized as follows. We give a brief introduction of the Molecular MAC protocol and its channel assignment problem in Section II. In Section III, the proposed scheme is presented in detail. In Section IV, performance studies are carried out through simulation results. Finally, we draw a conclusion in Section V.

\section{RELATED WORK}

This Section is designed to briefly specify the Molecular MAC protocol and then touch on certain problem relating to the channel allocation.

\section{A. Molecular MAC Protocol}

The IEEE 802.11 wireless network functions well in the infrastructure mode. In addition, it can provide a fair bandwidth to all users by slightly modifying a channel access method. Nevertheless, the IEEE 802.11 network may incur numerous problems on a multi-hop network. The Molecular MAC protocol expands the IEEE 802.11 network in order to transmit data packets on the multi-hop network effectively.

Since the IEEE 802.11 access method works well on a single-hop network, the Molecular MAC protocol divides a wireless mesh network into different spatially distributed atoms. Each atom uses a channel not used by other neighboring atoms. An atom is composed of one nucleus and several electrons, and a nucleus selects a channel to be used by its own atom. Any node within an atom's boundary plays a role as an electron and belongs to a neighboring atom. An electron directly communicates with its nuclei; however, since there is no direct link between electrons, a direct communication among them cannot be implemented. In addition, due to no direct link among nuclei, direct communications among them are also not possible. Therefore, the communication among neighboring electrons can be handled by nuclei while the communication among nuclei can be executed by neighboring electrons.

In the Molecular MAC protocol, each node is assigned with the role as a nucleus or an electron while each nucleus selects a channel to be used by its own atom. Accordingly, each node forms a shortest path-spanning tree to a gateway node linked to a wired Internet. After a tree is formed, the nodes with an evennumbered depth and an odd-numbered depth are assigned with the roles of a nucleus and an electron, respectively. After such roles are assigned, each nucleus requests its own electrons for channel information. Channel information includes a list of active channels and their activities. A list of active channels includes the numbers of active channels in the corresponding electrons' parent atoms. A channel activity is a parameter that is designed to indicate how many data packets are transmitted on each channel in the active channel list, which is expressed in the number of packets transmitted. Requested by a nucleus, each electron makes up a list of active channels, measures each channel's activities, and accordingly responds to the nuclei. Then, a nucleus receives certain responses from all neighboring electrons and accordingly selects a channel according to a subsequent rule. 1) After a list of active channels is received from all neighboring electrons, a channel is randomly chosen out of those non-active channels. 2) If every channel is currently occupied, a channel with the least activity is selected. Once a nucleus allocates a channel, its neighboring electrons use the channel. The corresponding electrons use all channels allocated by their atoms' nuclei.

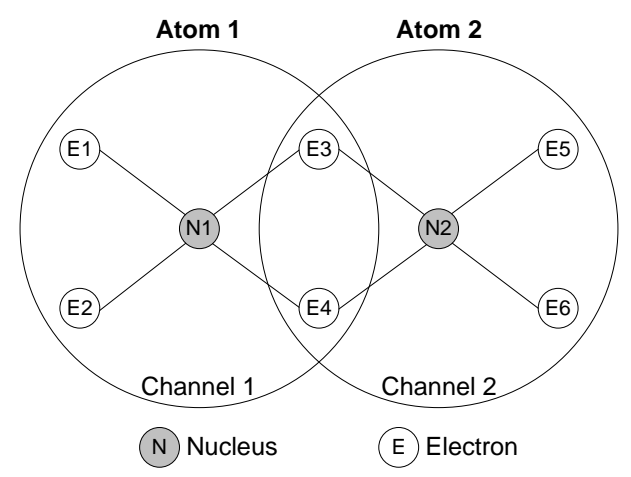

Fig. 3. Basic architecture of Molecular MAC protocol

Fig. 3 illustrates a fundamental structure of the Molecular MAC protocol. As shown in the figure, there are 2 atoms, 2 nuclei and 6 electrons. The atom 1 includes the nucleus $\mathrm{N} 1$ and the electrons (E1, E2, E3 and E4), and uses the channel 1. The atom 2 includes the nucleus $\mathrm{N} 2$ and the electrons (E3, E4, E5 and E6), and uses the channel 2. The electrons E3 and E4, shared by the two atoms, use both channel 1 and channel 2. If a neighboring atom's nucleus requests information for the allocation of a channel, the corresponding electrons transmit their channel activity information. Furthermore, the electrons $\mathrm{E} 1 / \mathrm{E} 2, \mathrm{E} 3 / \mathrm{E} 4$ and E5/E6 also transmit the active channel lists including the channel 1 , the channels $1 \& 2$ and the channel 2, respectively, to the nucleus. Once all channels are allocated, communications are processed as follows: As shown in the figure, when certain data are to be transmitted from E1 to E6, 
E1 transmits the data to $\mathrm{N} 1$ using the channel 1 while $\mathrm{N} 1$ transmits the date back to E3 or E4. Then, E3 or E4 transmits the data to N2 using the channel 2 while N2 transmits the data back to E6. The neighboring electrons, E1 and E2, do not directly communicate with each other but communicate via the nucleus N1.

\section{B. Channel Assignment Problem in the Molecular MAC Protocol}

There is a problem associated with a channel allocation scheme of the Molecular MAC protocol. The active channel list and channel activity information used by a nucleus for allocating a channel are not sufficient. If there are no electrons shared by neighboring atoms and if such atoms exist in the same interference range, the other atom's channel information cannot be identified. Therefore, an identical channel is allocated so that network performance can be deteriorated due to the interference. In addition, since each electron is located out of the other electron's transmission range, the number of packets transmitted cannot be properly measured, hence making the channel activity information inaccurate. As shown in the Fig. 3, because the atoms 1 and 2 share the electrons E3 and $\mathrm{E} 4$, one atom can identify the other atom's active channel when the nuclei N1 and N2 allocate a channel. In this way, an overlapped channel allocation can be avoided. That is, the nucleus N1 selects the channel 1 since it is aware that the channel 2 is currently occupied by the atom 2 . Similarly, the nucleus N2 is aware of the current occupation of the channel 1 by the atom 1 and thereby selects the channel 2 . However, as shown in the Fig. 4, because there is no electron directly linked between the atom 1 and the atom 2, actives channels occupied by the atoms cannot be identified. Therefore, the nuclei $\mathrm{N} 1$ and $\mathrm{N} 2$ may allocate the same channel. In the figure, the two atoms use the channel 1 .

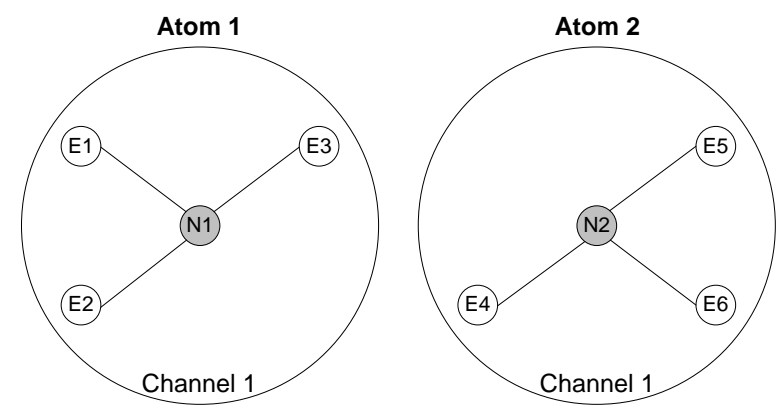

Fig. 4. Channel assignment problem

\section{INTERFERENCE AWARE CHANNEL ASSIGNMENT (IACA) SCHEME}

In this paper, a new method to resolve such issues involved with the allocation of a channel in the Molecular MAC protocol is proposed. Although the proposed scheme naturally adopts a fundamental operation principle of the Molecular MAC protocol, it modifies a channel allocation method.

Based on the scheme proposed in this paper and with the corresponding interference taken into account, a list of active channels is formed while their activities are measured. Based on this information, a nucleus allocates a channel. In the Molecular MAC protocol, the activity information, expressed in the number of packets transmitted, is applied. However, the proposed scheme utilizes the collision probability information. Although the utilization of a channel is clearly a possible alternative, it is not used since it differs as per an access method (basic or RTS/CTS) or size of data packets. Because a collision probability is independent of an access methods or size of data packets, it is more efficient to apply the probability values. Collision probability is used as a simple and effective estimate of the channel contention level.

When a channel is to be allocated in the Molecular MAC protocol, a nucleus receives a list of active channels from all neighboring electrons and then randomly selects a channel out of those non-active channels. If every channel is currently occupied, a channel with the least activity is selected. However, the proposed scheme uses the active channel list \& collision probability information, identifies all non-active channels and then randomly selects a channel from those non-active channels. In addition, if every channel is currently occupied, a channel is chosen based on the collision probability information.

Similar to the Molecular MAC protocol, the proposed scheme first selects a non-active channel using a list of active channels. Then, it uses the collision probability information in order to identify any overlapped channel used by another atom and accordingly allocates a new channel.

In order to measure the collision probability, both a nucleus and an electron manages the number of packets ( Packet $_{\text {sent }}$ )

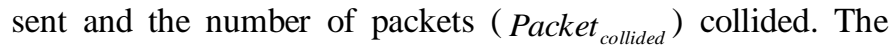
nucleus manages these values for each electron. Packet ${ }_{\text {sent }(i, j)}$

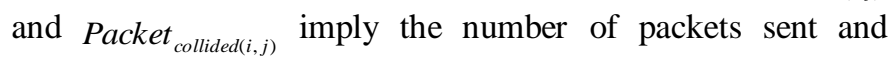
collided, respectively, for the node $\mathrm{i}$ and the node $\mathrm{j}$. In the variable $(\mathrm{i}, \mathrm{j})$, one represents a nucleus and the other denotes an electron. Based on these values, a collision probability $\left(P_{\text {coll }(i, j)}\right)$ can be computed as follows.

$$
P_{\text {coll }(i, j)}=\frac{\text { Packet }_{\text {collided }(i, j)}}{\text { Packet }_{\text {sent }(i, j)}}
$$

Note that $P_{\text {coll }(i, j)}$ is always in the range of $[0,1]$ and estimating the value is simple without any additional overheads or costs. If a station does not receive an ACK for the basic method or a CTS for the RTS/CTS method, the station assumes the packet is collided. The station does not count at all any packets from other stations.

Collision probability has to be maintained and updated by each node (nucleus and electrons in an atom). We assume collision probability is updated per each update period (UpdateTime) expressed in time. Each node counts the number of packets sent $\left(\operatorname{sent}_{n}\right)$ and collided $\left(\operatorname{coll}_{n}\right)$ during the nth update period and then calculates instantaneous collision probability ( $\left.P_{\text {coll }}^{\text {current }}\right)$ using (1). From this value, average 


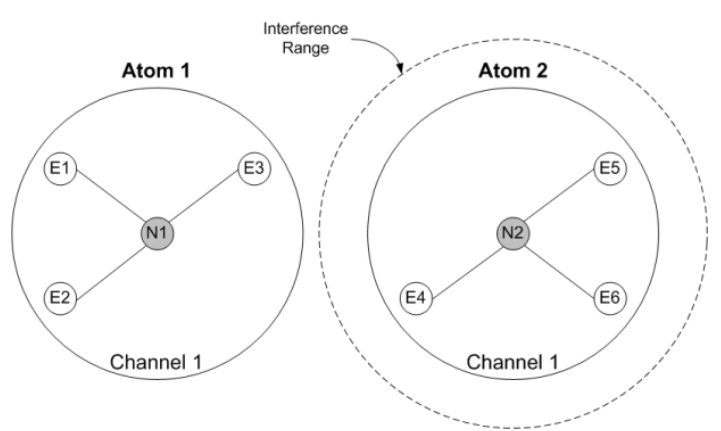

a) Atom 1 is not within the interference range of Atom 2

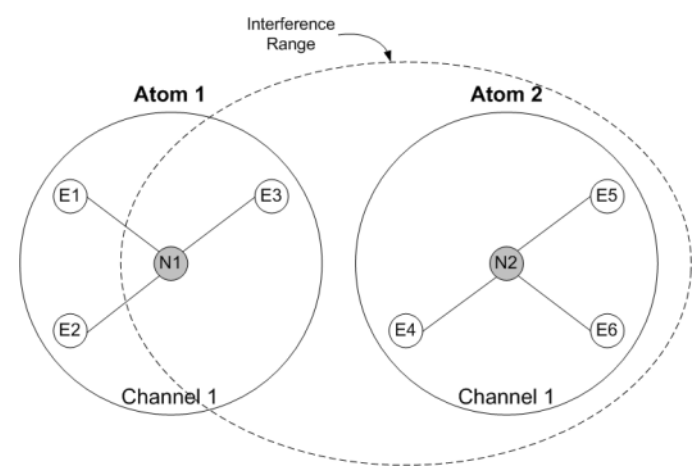

c) Nucleus and some electrons in Atom 1 is within the interference range of Atom 2

Fig. 5. Four cases according to the interference range of atom

collision probability $\left(P_{\text {coll }}^{n}\right)$ is approximated by exploiting a moving averaging window:

$$
P_{\text {coll }}^{n}=\alpha \cdot P_{\text {coll }}^{n-1}+(1-\alpha) \cdot P_{\text {coll }}^{\text {current }}
$$

where $P_{c o l l}^{n-1}$ is the average collision probability at the end of the (n-1)th period and $\alpha$ is a smoothing factor.

An electron calculates a collision probability and periodically transmits it to a nucleus.

As illustrated in the four cases of Fig. 5, the nucleus uses the collision probability and allocates a new channel in order to avoid an overlapped channel allocation.

Firstly, if the atom 1 is not covered by the interference range of the atom 2 , the atom 1 is not affected by the atom 2; therefore, the performance of the atom 1 is not deteriorated, and it is okay to continue to utilize the current active channel. That is, there is no need to consider a collision probability.

Secondly, if part of the electrons included in the atom 1 is covered by the interference range of the atom 2, a collision probability measured by the electrons is greater than that measured by the nucleus. Hence, if the two collision probabilities differ by more than a threshold value, it implies that the current active channel is also occupied by other neighboring atoms; therefore, a new channel is allocated. However, if the difference is negligible, the effect of the

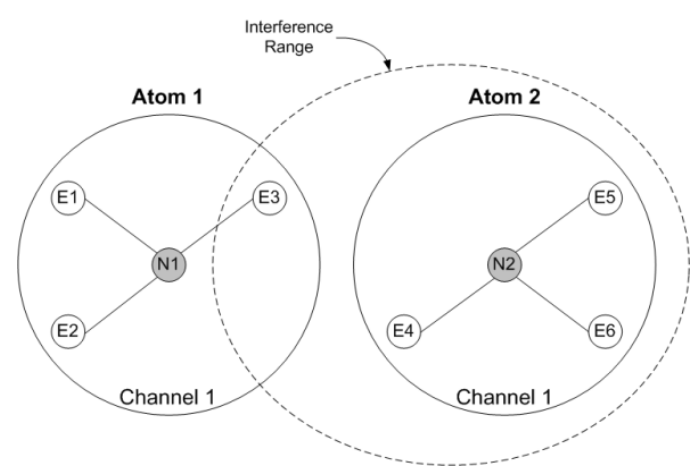

b) Some electrons in Atom 1 is within the interference range of Atom 2

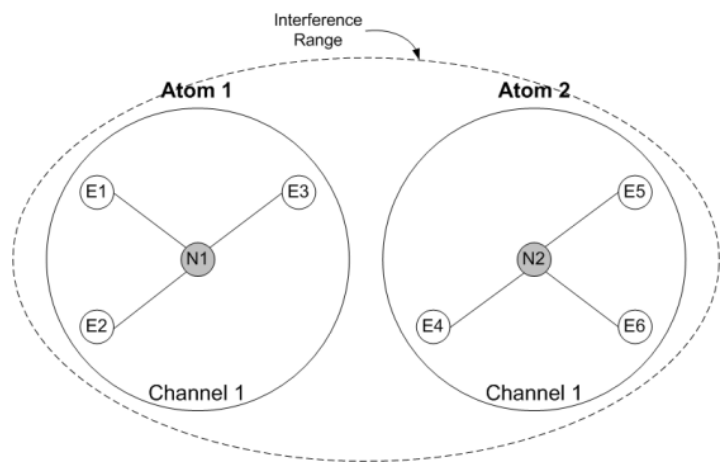

d) Nucleus and all electrons in Atom 1 is within the interference range

interference is minimal, therefore making the utilization of the current channel feasible.

Thirdly, if a nucleus and some electrons of the atom 1 are covered by the interference range of the atom 2, a collision probability measured by the nucleus is similar to that measured by the electrons.

Therefore, similar to the second case, a new channel cannot be allocated. However, this issue can be cleared by using an electron of the atom 1 not covered by the interference range of the atom 2 and factoring in a collision probability measured by the electron. A collision probability measured by the noninterfered electron is less than that measured by the nucleus. That is, if there is any difference between the two collision probabilities, it implies that one atom is affected by another atom's interference; therefore, a new channel is allocated.

For the second and the third cases, if the collision probability measured by the nucleus differs from that measured by the electron by more than a threshold value, a new channel is allocated. That is, a new channel can be allocated if the following formula is satisfied. In the variable $(i, j)$, one represents a nucleus and the other denotes an electron.

$$
P_{\operatorname{coll}(i, j)}>P_{\operatorname{coll}(j, i)}+\beta
$$

where, $\beta$ is a threshold value for changing channel. 
Finally, if a nucleus and all electrons of the atom 1 are covered by the interference range of the atom 2 , those collision probabilities measured by the nucleus and the electrons is similar; therefore, a new channel cannot be allocated because the (3) cannot be satisfied. Still, the network performance is deteriorated due to the impact by the atom 2 . In this case, the nucleus factors in a total collision probability $\left(P_{\text {total_coll }}\right)$.

$$
P_{\text {total_coll }}=\frac{\sum_{j} \text { Packet }_{\text {collided }(i, j)}}{\sum_{j} \text { Packet }_{\text {sent }(i, j)}}
$$

where, $i$ represents a nucleus while $j$ denotes an electron.

In order to show the effect of the number of nodes on collision probability, we simulated an IEEE 802.11a network with transmission rates of $54 \mathrm{Mbps}$ for data packets and of 6 Mbps for control packets such as RTS, CTS and ACK, respectively. We assume that each node always has packets to transmit, and all packets are of the same size. This environment is extreme case. In Fig. 4, collision probability for the IEEE 802.11 DCF gets higher as the number of stations becomes larger. This is from the fact that all nodes always try to access the channel and make collisions with one another.

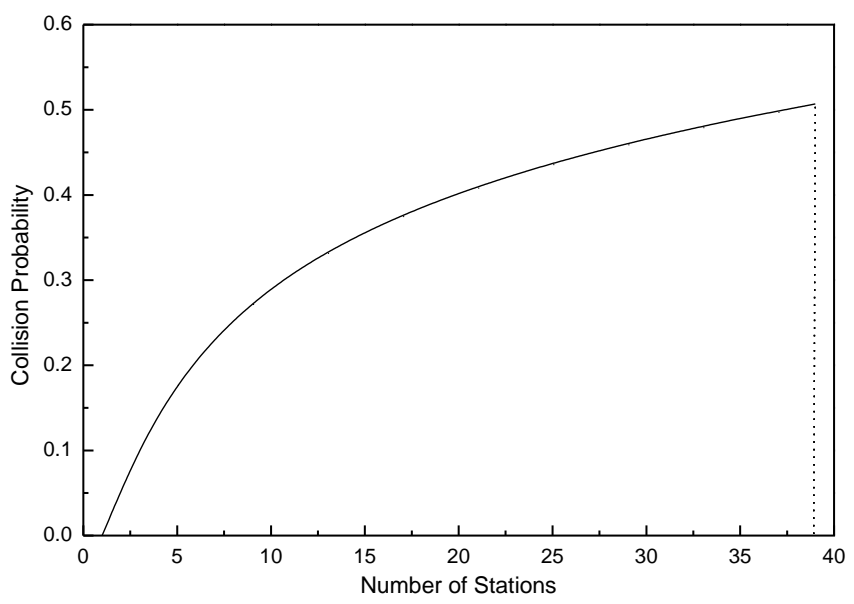

Fig. 6. Collision probability according to the number of nodes

Fig. 6 shows such collision probabilities resulted from the worst-case scenario. That is, any normal environment other than that of Fig. 6 generates better results. Therefore, a specific value based on such results provides an upper bound for a collision probability.

A nucleus determines whether to allocate a new channel using this value. For such determination, the nucleus manages the corresponding collision probabilities on a table-form basis. The nucleus is well aware of the number of electrons included in its own atom. Then, the nucleus compares the total collision probability computed in (4) with the value resulted from Fig. 6 and then determines whether to allocate a new channel. If the total collision probability is greater than that in the collision probability table by more than a threshold value, a new channel is allocated.

$$
P_{\text {total_coll }}>P_{\text {coll }, n}+\gamma
$$

where, $P_{\text {coll }, n}$ is the collision probability for $\mathrm{n}$ electrons in the table and $\gamma$ is a threshold value for changing channel.

If there is any non-active channel, a nucleus selects a channel based on the procedures mentioned above. However, if every channel is currently occupied, the nucleus selects a channel with the lowest collision probability.

\section{Simulation Results}

In this section, we discuss the simulation results of the proposed IACA scheme. To study the performance of the IACA scheme, we have implemented it. We compare them to the results of the Molecular MAC protocol. We simulated an IEEE 802.11a network with transmission rates of $54 \mathrm{Mbps}$ for data packets and of $6 \mathrm{Mbps}$ for control packets such as RTS, CTS and ACK, respectively. Nodes use a network interface with the transmission range of 10 units and the interference range of 22 units.

The spanning tree construction proceeds as follows. First, the network elects a gateway node, which is connected to the wired Internet. Then, the other nodes construct a spanning tree rooted at the gateway node. We place the gateway node on the top-left corner and randomly the other nodes in a simulation topology.

Main performance metrics of interest are the number of atoms, the number of assigned channels and the number of neighboring atoms. The number of atoms indicates the number of total atoms produced in a spanning tree of the simulation topology. In addition, the number of assigned channels is the number of such channels allocated to nuclei in the simulation topology. That is, it is the minimum number of channels required to serve every node in the simulation topology. The number of neighboring atoms represents the number of those neighboring atoms when an atom's nucleus selects a channel. Because each atom occupies one channel, the number of neighboring atoms means the number of active channels in other neighboring atoms. All simulation results are averaged over ten simulations.

Table I shows the simulation results (number of neighboring atoms, number of assigned channels) when there are 50 nodes. For the Molecular MAC protocol, since each nucleus factors in 3 neighboring atoms on average and accordingly allocates a channel, approximately 4.1 channels are required to serve every node in the simulation topology. However, because the proposed IACA scheme takes 8.1 neighboring atoms into account, roughly 8.7 channels are needed. The Molecular MAC protocol identifies the number of neighboring atoms with help from those electrons in the transmission range of a nucleus and accordingly allocates a channel. However, the proposed scheme expands the scope up to the interference range; therefore, it can identify more neighboring atoms and accordingly allocate a channel. Because the proposed scheme considers 2.7-times more neighboring atoms than the Molecular MAC protocol and accordingly allocate a channel, about 2.1-times more channels can be allocated. Furthermore, in the Molecular MAC protocol, some 
neighboring atoms still allocates the same channel, hence deteriorating the performance of a network. On the other hand, the proposed scheme prevents the allocation of the same channel by those neighboring atoms in the interference range and thereby enhances network performance.

TABLE I. SIMULATION RESULTS FOR 50 NODES

\begin{tabular}{l|c|c}
\hline & $\begin{array}{c}\text { Molecular MAC } \\
\text { protocol }\end{array}$ & IACA scheme \\
\hline Number of neighboring atoms & 3.0 & 8.1 \\
Number of assigned channels & 4.1 & 8.7 \\
\hline
\end{tabular}

Figs. 7, 8 and 9 show how network performance is changed as the number of nodes increases. In particular, Fig. 7 indicates how the number of those atoms produced in the simulation topology linearly increases in proportion to the number of nodes. If the number of nodes equals to 30 , about $27 \%$ of total nodes become nuclei. As the number of nodes increase, such percentage declines. For example, if the number of nodes is 70 , approximate $18 \%$ of total nodes become nuclei. Fig. 8 depicts the number of those neighboring atoms to be considered when each nucleus allocates a channel. In the Molecular MAC protocol, because the information about neighboring atoms is collected from those electrons in the transmission range of a nucleus, the corresponding result value is quite low. On the other hand, the proposed scheme further considers the interference range and accordingly collects the information about neighboring atoms; therefore, the corresponding result value is relatively higher. As the number of nodes increases, the number of neighboring atoms for each nucleus accordingly increases. Fig. 9 shows the number of those channels allocated to serve every node in the simulation topology. This result is similar to that of Fig. 8. As the number of neighboring atoms increases, the number of channels allocated accordingly increases since other channels are allocated to avoid any overlapping. Compared to the Molecular MAC protocol, the IACA scheme requires 1.9-times more and 2.1-times more channels if the number of nodes is 30 and 70 , respectively. Based on such results, an identical channel is not allocated to neighboring atoms, therefore enhancing the performance of a network.

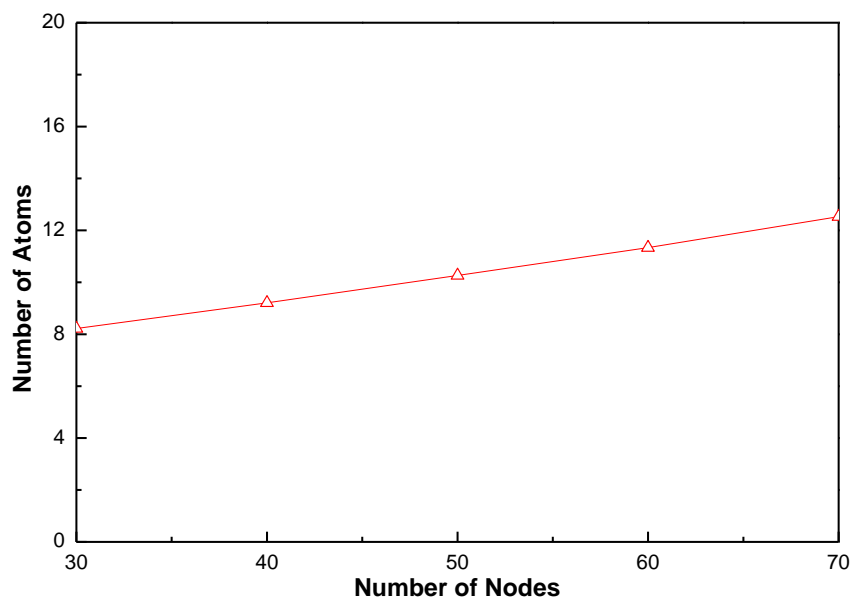

Fig. 7. Number of atoms according to the number of nodes

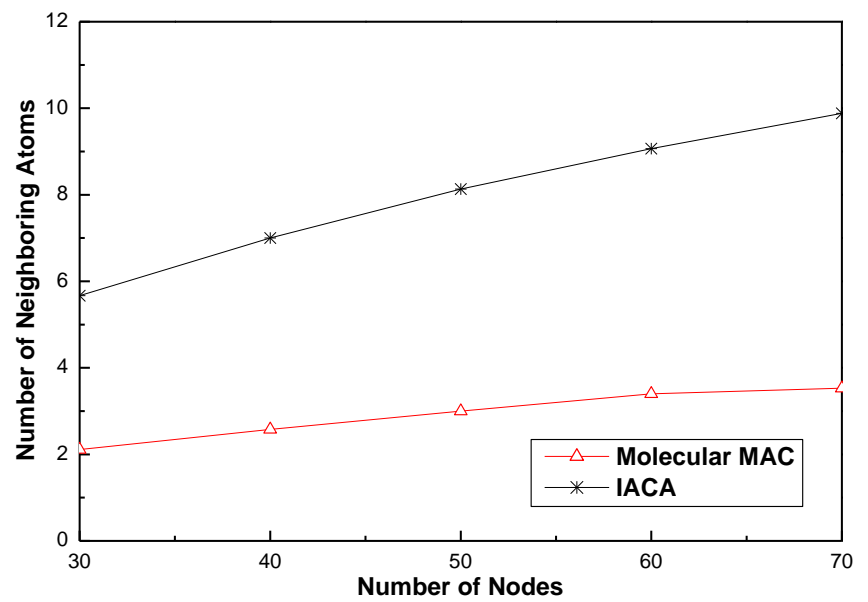

Fig. 8. Number of neighboring atoms according to the number of nodes

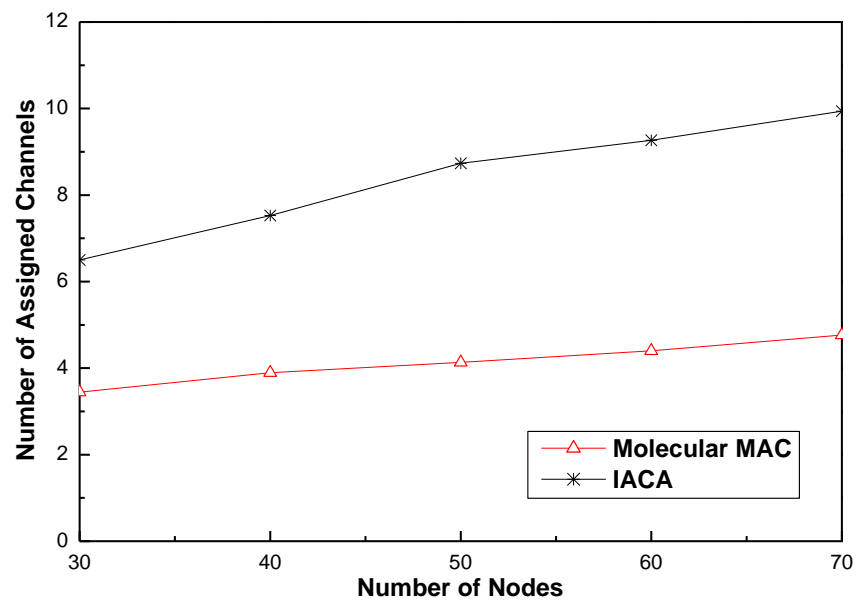

Fig. 9. Number of assigned channels according to the number of nodes

\section{CONCLUSION}

A wireless mesh network is a promising wireless technology for numerous applications. In mesh networks with a single channel, the functionalities of nodes are performed on the same channel. Therefore, network performance decreases. In order to increase the performance, several MAC protocols have been proposed based on multiple channels. Molecular MAC protocol was proposed by adopting a molecular analogy. In this protocol, a nucleus assigns a channel for its atom with the assistance from electrons in the same atom. It is not able to recognize the existence of those atoms in the interference range. Therefore, allocating the same channel to neighboring atoms results in the deterioration of network performance. In order to resolve this problem, we propose a novel distributed interference aware channel assignment scheme. The proposed scheme is somewhat similar to the Molecular MAC protocol method in that a channel is selected by the assistance of electrons. Then, a collision probability is applied to allocate a new channel in order to recognize any interference and avoid any overlapped channel allocation. Simulation results show that the proposed scheme was verified that different channels could be allocated to those neighboring atoms in the interference range. 


\section{REFERENCES}

[1] I.F. Akyildiza, X. Wang, and W. Wang, "Wireless mesh networks: a survey," Elsevier Computer Networks, vol. 47, no. 4, pp. 445-487, 2005.

[2] A. Iyer and C. Rosenberg, "Understanding the key performance issues with MAC protocols for multi-hop wireless networks," Wireless Communications and Mobile Computing, vol. 6, no. 6, pp. 745-760, 2006.

[3] H. Zhai, X. Chen, and Y. Fang, "Alleviating intra-flow and inter-flow contentions for reliable service in mobile ad hoc networks," in Proceedings of IEEE MILCOM, vol. 3, pp. 1640-1646, Nov. 2004.

[4] M. Karaliopoulos, T. Spyropoulos, and B. Plattner, "Interference-aware routing in wireless multihop networks," IEEE Transactions on Mobile Computing, vol. 10, no. 5, pp. 716-733, May 2011.

[5] Y. Kim, D. Jung, Y. Kim, S. Choi, and J. Ma, "Efficient interferenceaware channel allocation in multi-radio wireless mesh setworks," in Proceedings of International Conference on Advanced Communication Technology (ICACT 2012), pp. 920-925, 2012.

[6] P.B.F. Duarte, Z.M. Fadlullah, A.V. Vasilakos, and N. Kato, "On the partially overlapped channel assignment on wireless mesh network backbone: a game theoretic approach," IEEE Journal on Selected Areas in Communications, vol. 30, no. 1, pp. 119-127, Jan. 2012.

[7] A. Subramanian, H. Gupta, S. R. Das, and J. Cao, "Minimum interference channel assignment in multi-radio wireless mesh networks," IEEE Transactions on Mobile Computing, vol. 7, no. 12, pp. 1459-1473, Dec. 2008.

[8] F. Theoleyre, B. Darties, and A. Duda, "Assignment of roles and channels for a multichannel MAC in wireless mesh networks," in Proceedings of 18th International Conference on Computer Communications and Networks (ICCCN 2009), pp. 1-6, Aug. 2009.

[9] B. Darties, F. Theoleyre, and A. Duda, "A Divide-and-conquer scheme for assigning roles in multi-channel wireless mesh networks," in
Proceedings of IEEE 34th Conference on Local Computer Networks (LCN 2009), pp. 277-280, Zurich, Switzerland, Oct. 2009.

[10] D. Abdelali, F. Theoleyre, A. Bachir, and A. Duda, "Neighbor discovery with activity monitoring in multichannel wireless mesh networks," in Proceedings of IEEE Wireless Communications and Networking Conference (WCNC 2010), pp. 1-6, Apr. 2010.

[11] M. Nassiri, F. Theoleyre, M. Heusse, and A. Duda, "Molecular architecture for autonomic wireless mesh networks," In Proceedings of the ACM student workshop - CoNEXT 2007, Dec. 2007.

[12] F. Theoleyre, M. Heusse, and A. Duda, "Molecular MAC for multichannel wireless mesh networks," in Proceedings of IEEE 6th International Conference on Mobile Adhoc and Sensor Systems (MASS 2009), pp. 110-119, Oct. 2009.

[13] S. Pollak, V. Wieser, and A. Tkac, "A channel assignment algorithm for wireless mesh networks with interference minimization," in Proceedings of Wireless and Mobile Networking Conference (WMNC 2012), pp. 1721, 2012.

[14] K.N. Ramachandran, E.M. Belding, K.C. Almeroth, and M.M. Buddhikot, "Interference-aware channel assignment in multi-radio wireless mesh networks," in Proceedings of IEEE INFOCOM, pp. 1-12, Apr. 2006.

[15] K. Xing, X. Cheng, L. Ma, and Q. Liang, "Superimposed code based channel assignment in multi-radio multi-channel wireless mesh networks," in Proceedings of ACM MobiCom, pp. 15-26, 2007.

[16] H. Skalli, S. Ghosh, S. Das, L. Lenzini, and M. Conti, "Channel assignment strategies for multiradio wireless mesh networks: issues and solutions," IEEE Communications Magazine, vol. 45, no. 11, pp. 86-95, Nov. 2007.

[17] S. Avallone, G. Stasi, and A. Kassler, "A Traffic-aware channel and rate re-assignment algorithm for wireless mesh networks," IEEE Transactions on Mobile Computing, in press, 2013. 